\title{
A Method for Estimating Costs and Benefits Of Space Assembly and Servicing By Astronauts and Robots
}

\author{
Lloyd R. Purves \\ NASA \\ Goddard Space Flight Center \\ Greenbelt, MD 20771 \\ 301-286-4207 \\ lloyd.r.purves.1@gsfc.nasa.gov
}

\begin{abstract}
One aspect of designing future space missions is to determine whether Space Assembly and Servicing (SAS) is useful and, if so, what combination of robots and astronauts provides the most effective means of accomplishing it. Certain aspects of these choices, such as the societal value of developing the means for humans to live in space, do not lend themselves to quantification. However, other SAS costs and benefits can be quantified in a manner that can help select the most cost-effective SAS approach.
\end{abstract}

Any space facility, whether it is assembled and serviced or not, entails an eventual replacement cost due to wear and obsolescence. Servicing can reduce this cost by limiting replacement to only failed or obsolete components. However, servicing systems, such as space robots, have their own logistics cost, and astronauts can have even greater logistics requirements. On the other hand, humans can be more capable than robots at performing dexterous and unstructured tasks, which can reduce logistics costs by allowing a reduction in mass of replacement components. Overall, the cost-effectiveness of astronaut SAS depends on its efficiency; and, if astronauts have to be wholly justified by their servicing usefulness, then the serviced space facility has to be large enough to fully occupy them.

\section{TABLE OF CONTENTS}
1. INTRODUCTION
2. BACKGROUND
3. APPROACH
4. EXAMPLES
5. CONCLUSIONS
6. REFERENCES
7. BIOGRAPHY

\section{INTRODUCTION}

Significant challenges have been associated with Space Assembly and Servicing (SAS) since almost the beginning of the space age. However, these challenges have been more associated with the cost effectiveness of SAS than its technology. With respect to SAS cost effectiveness, some questions that have long been considered important are when to use either astronauts or robots, and whether there is some productive way to use the two together.

This paper presents the results of an initial study to address these questions by using a quantitative model of the associated costs and benefits. Because this initial effort was limited, it uses simple assumptions. Despite its simplicity, the model appears capable of deriving some important SAS guidelines. Should sufficiently precise answers be desired to make credible decisions about specific missions, it appears that a similar approach could be used, but with more complex and realistic representations of costs and benefits.

\section{BACKGROUND}

To provide some background and basis for the quantitative model, this paper will first summarize the:

- Definition of SAS

- Rationale for SAS

- Options for accomplishing SAS

- History of SAS

\section{Definition of Space Assembly and Servicing}

Conceptually, space assembly and servicing are fairly simple and quite similar. Assembly consists of attaching together in space the various components that make up some space facility. Servicing largely consists of replacing certain components on an existing space facility. Replacing a given component generally means detaching it and then attaching its replacement. Thus any system for attaching and detaching components in space can potentially provide space assembly and servicing.

\section{Rationale for Space Assembly and Servicing}

The rationale for space assembly and servicing is likewise basically simple. If a space facility is desired that is 100 large or too heavy for one launch vehicle then it has to be 
assembled in space. Potentially, space assembly could also be justified if it were effectively less expensive than some sufficiently complex set of deployment mechanisms.

Space servicing is potentially important because all space components in time lose value because of failure, degradation, or obsolescence. If, as usually happens, some components on a space facility lose value faster than others, then it can be more economical to replace just these components rather than the whole facility.

\section{Options for Space Assembly and Servicing}

Space assembly and servicing starts to get complex when one looks at the options, all of which must be considered in order to design the most cost effective space mission. Some of the major options include:

1. Avoid space assembly and servicing altogether by making the facility small enough and replacing all of it when, for whatever reasons, its utility drops below some threshold. This is in fact how most space missions have been and are still designed.

2. If possible, achieve the capability of a large space facility by flying a set of smaller facilities in formation, as is proposed for some future missions such as the Terrestrial Planet Finder (TPF), which is envisioned as a set of formation flying telescopes acting together as an interferometer. With this approach, assembly does not require physical attachment; nor does servicing require detachment and reattachment, as long as the components to be replaced are defined as the individual facilities flying in formation.

3. Use rendezvous and docking (along with undocking and departure) to provide the assembly and disassembly functions. This was the means for the assembly of certain modules of the International Space Station (ISS). The Russian Control and Service modules were joined using automated rendezvous and docking, and the US node was attached to ISS using a manually controlled Shuttle rendezvous and docking.

4. Use Astronauts. A good example is the Hubble Space Telescope (HST) on which astronauts have performed the vast majority of servicing tasks. While the HST servicing tasks were performed using Extra-Vehicular Activity (EVA), astronauts can also use Intra-Vehicular Activity (IVA) for SAS.

5. Use Robots. Examples of this are more complex to define as the Remote Manipulator System (RMS) on the Shuttle is sometimes described as a robot arm and sometimes as a crane. It is probably most accurately described as tele-operated (i.e. human controlled) manipulator of fairly large objects. It can be operated and has been tested as an autonomous robot (i.e. computer controlled), but the risks of this mode are considered greater than its labor saving value, so it not known to have been used operationally. In its teleoperated mode the RMS has been used extensively on many Shuttle missions, particularly the HST servicing missions.

6. Combinations of any of the above. SAS on the ISS is a good example as it employs a combination of options 3 , 4 and 5.

\section{History of Space Assembly and Servicing}

In the early years of the space program the main objective was to successfully carry out groundbreaking missions that were quite limited compared to those of today and for which SAS was not applicable. The most important consequence of the launch of Sputnik in 1957 was the immense boost it gave the USSR in its international standing. Given the cold war competition between the US and the USSR, this created a comparably immense challenge for the US. When in 1961 the USSR also became the first country to put a human, Yuri Gargarin, into space, the geo-political challenge for the US became even greater.

The consequence of these USSR "space spectaculars" was to set the US on the course of the immensely expensive Apollo program to land the first humans of the moon. Basically Apollo was a throwaway system that was neither assembled nor serviced in space. However, space rendezvous and docking was used two times on each lunar landing mission to connect and reconnect the command and landing modules. It is interesting to note that serious consideration was given to first assembling a space station in LEO and then using it to assemble a lunar vehicle in orbit, but this approach was eventually rejected as taking too long

By the time Apollo's goal was successfully accomplished in 1969, the US had surpassed the accomplishments of the USSR in space by such an extent that the space race had effectively come to an end. This left the US with a manned space infrastructure for which there was no need important enough to justify its cost. However, the US did not have the option of wholly abandoning a human space effort, in part because the USSR continued to slowly advance its own more modest human space program directed toward a space station in low earth orbit. It should be noted that these USSR Soyuz space stations used space rendezvous and docking for assembly, and astronauts for servicing.

It was at this point that the issue of space assembly and servicing became important in the US. It was decided that the cost of a US human space program could be better justified if astronauts could support other space efforts, such as by launching, and possibly assembling and servicing the communication and observation satellites used for military, 
scientific, environmental and commercial applications.

Consequently, and at considerable cost, the US Space Shuttle was developed to support SAS by providing such capabilities as rendezvous and docking, an airlock and space suits for EVA, the RMS to manipulate large objects, and an overall ability to support more than a half dozen astronauts for over a week of activity in space. The Shuttle was also intended to lower the cost of all (SAS and non-SAS) space missions, by being mostly reusable and by providing a large payload volume and mass capacity

Despite the setback of the total loss in 1986 of the Space Shuttle Challenger and all of its crew, the Shuttle has used its capabilities over the last decade to successfully support on the order of 10 SAS missions for the Hubble Space Telescope (HST) and the International Space Station (ISS). Yet, the outcome has been that HST servicing is due to be terminated in about 2004 after about 3 more missions, and, outside of the ISS, no other existing or planned space mission intends to employ SAS.

Basically, and partly as a result of the Challenger disaster, current thinking is that the cost and constraints of making the Shuttle sufficiently safe for astronauts have priced it out of the reach of space missions that do not specifically need astronauts. There also appears to be a general consensus that SAS, despite it promise, is not cost effective, at least outside of the ISS program.

The are multiple reasons why the extensive, successful, and continuing SAS activities on the ISS program are not considered proof that SAS would be cost-effective on other missions. A primary one was that the ISS program was not based on providing a more cost effective way of accomplishing things that other missions could do. In fact, like the Apollo moon program, a major impetus for the ISS was political, specifically a perceived need to provide a nonmilitary space project for the Russian space establishment, which was largely unemployed after the collapse of the USSR and might otherwise have sold it skills to wealthy nations that were antagonistic to the US. Another reason was that there was no realistic choice but to use SAS on the ISS. Being too large for any single LV the ISS has to be assembled. Being too expensive to replace, it also has to be serviced.

This brings us to the current situation where little quantitative analysis exists to indicate how different SAS approaches (including not doing SAS) could affect mission costs. It is an initial and simplified approach to the kind of analysis that is presented below.

\section{APPROACH}

The approach for evaluating the cost of various options for Space Assembly and Servicing is based on the following simplified assumptions

1. A space facility has an objective of some value that it accomplishes over its mission lifetime.

2. During the course of mission components fail or degrade at constant average rate. Also, during the course of the mission technology improves, which causes components to become obsolete at a constant average rate.

3. After its mission is accomplished the facility is replaced by some follow-on facility that has more capabilities and commensurately greater objectives.

4. The average cost of space flight equipment is proportional to its mass. The associated launch and operations costs are also assumed to be proportional to the mass of the space flight hardware. Naturally items like detectors have a much greater cost per unit mass than, say, aluminum structure. So what this statement is effectively saying is that the proportion of these different cost-per-unit-mass items stays relatively constant from one mission to the next.

5. Therefore, whatever approach allows a given mission to be accomplished with the least mass of space flight hardware will provide the lowest cost solution.

\section{EXAMPLES}

How these assumptions could work out in practice can be illustrated by using the example of a space telescope similar to HST. For purposes of comparison HST has a mass of $12,000 \mathrm{~kg}$, a Low Earth Orbit (LEO), and an expected mission life of 20 years. HST is expected to remain completely functional for the approximately 3-year period between Shuttle servicing missions. Because HST is statistically expected to have component failures during these periods, it has enough redundancy that anticipated failures should not degrade its performance. HST actually becomes more capable with servicing when some HST instrumentation is replaced with improved versions.

For purposes of illustration, let us consider a HST-like telescope [1] called the Reference Space Telescope (RST) and assume that it has a mass of $10,000 \mathrm{~kg}$ and needs an average of one component replaced per year per $1000 \mathrm{~kg}$ of mass, due to failure, degradation or upgrading. This comes out to 10 component replacements per year. Therefore, over its 20-year lifetime some 200 new components will be supplied. At the end of 20 years, we assume its basic 
architecture (for example aperture of the primary mirror) is so obsolete that total replacement is better than continued servicing.

The concept that the number of annual component replacements is proportional to mass is important and has different ramifications for different SAS approaches. Basically what is being assumed is that the number of components on a space facility is proportional to its mass, and that a constant percentage of the total number of components needs to be replaced each year. However, the mass of the replacement component depends on the servicing scenario. For example, the HST has had a number of gyro failures, most of which were due to the failure of a tiny wire whose mass is measured in grams. However, EVA servicing does not replace the wire. Instead, a module containing two whole gyros and having a mass of some tens of $\mathrm{kg}$ is replaced. Conceivably, if the Shuttle had the right equipment on board, the astronaut could go back into the Shuttle and just replace the wire, thereby essentially eliminating the need to use any mass for this particular servicing operation.

Let us define the benefit of the RST as having a scientific return of 1000 in its first year. For this study the figure of 1000 is arbitrary, but it could be related or at least proportional to the number of peer-reviewed papers that result from the first year of RST operations. Let us also assume that 20 years of equipment upgrades leaves it twice as capable at the end of its life as it was at the beginning. Assuming it improves at a roughly constant rate due to equipment upgrades, it has an average annual scientific return of 1500 over its lifetime, which results in a Lifetime Scientific Return (LSR) of 30,000.

Given that RST cost is proportional its effective mass, and that its value can be measured in LSR units, then the most cost effective RST mission is the one with the highest LSR/kg, which will be defined as its Figure of Merit (FOM).

Described below are 12 SAS scenarios selected to illustrate major options and major ways of changing the FOM. All are summarized in Table 1.

\section{Scenario 1 (No SAS)}

The baseline or reference mission will be one that produces an LSR of 30,000 without SAS. For purposes of illustration, let us assume that the RST can be given enough additional redundancy and margin to be reliably expected to last 10 years instead of 3 , but at the cost of increasing its mass $50 \%$, i.e. the non-serviceable RST will have a mass of $15,000 \mathrm{~kg}$. At the end of 10 years it will be replaced by an improved RST, which also has a mass of $15,000 \mathrm{~kg}$ and a 10 -year design life, but a LSR of 2000 due to technology improvements over the 10-year interval. The two non- serviceable RST's will therefore produce the same LSR of 30,000 , but will also entail the development of $30,000 \mathrm{~kg}$ of spaceflight hardware, which gives a FOM of $1 \mathrm{LSR} / \mathrm{kg}$.

\section{Scenario 2 (SAS using EVA from the STS)}

This scenario basically reflects the HST mission. A key assumption is that, with astronaut EVA servicing, the average mass of a module containing a RST replacement component is $25 \mathrm{~kg}$. Therefore, with 200 components replaced over 20 years, the RST gets $5000 \mathrm{~kg}$ of new modules over its lifetime. This means the RST program has to develop $15,000 \mathrm{~kg}$ of spaceflight hardware to get the 20 year lifetime and the LSR of 30,000 .

If we assume that the RST is serviced by 6 dedicated Shuttle missions (one every three years for 18 years) and that the human space program covers all of the astronaut related servicing costs in including Shuttle launches, then the cost for the RST program stays proportional to just the $15,000 \mathrm{~kg}$ of hardware.

This approach yields a FOM of $2 \mathrm{LSR} / \mathrm{kg}$, which is twice as good as the one for the non-serviceable RST; however the costs to the human space program are ignored.

\section{Scenario 3 (SAS using EVA from a Space Station)}

Using the Shuttle, it is difficult to separate EVA cost from STS launch costs. To get a clearer picture of what astronaut EVA costs might be, let us assume a Reference Space Station (RSS), which is similar to the current ISS, but optimized for servicing the RST.

The actual ISS (circa 2001) has an approximate mass of 100 metric tons and three permanent crewmembers. To simplify calculations we will assume a RSS of 100 metric tons, which is $100,000 \mathrm{~kg}$, and a crew of two, who together require $20,000 \mathrm{~kg}$ of logistics annually. Assuming the RSS is in the same orbit as the ISS, this amount of annual logistics comes to about two STS missions per year. Thus, over a 20 -year period, the RSS has a total mass requirement of $500,000 \mathrm{~kg}$.

The RSS will be designed so that only one astronaut will be required to maintain the RSS and the other can be dedicated to RST servicing or other tasks. The RSS will also include a space tug to bring one or more co-orbiting RST's over for servicing. For this scenario we take the RSS as a provided facility and assume that the RST only pays for astronaut logistics to cover the time spent servicing the RST.

We will furthermore assume that each RST module replacement takes an average of one day of RSS astronaut time, which covers not only the EVA time, but also training, etc. Therefore, replacing 200 modules represent 200 work days, or about 1 -astronaut work year, assuming they have weekends off. Therefore, to have 1 year of RSS astronaut 
time for the RST would require the RST program to pay for $20,000 \mathrm{~kg}$ of RSS logistics supplies. The means that the RST program would have to pay for $10,000 \mathrm{~kg}$ of RST, $5,000 \mathrm{~kg}$ of replacement modules, and $20,000 \mathrm{~kg}$ of RSS astronaut logistics, for a total of $35,000 \mathrm{~kg}$ to get the LSR of 30,000 .

The FOM is therefore $0.86 \mathrm{LSR} / \mathrm{kg}$, which is worse than for non-serviceable RST.

\section{Scenario 4 (RSS IVA servicing of the RST)}

Let us next look at a SAS scenario where we try to maximize astronaut efficiency and effectiveness. One promising way of accomplishing this will be to let the astronaut perform IVA rather than EVA, which should result in more dexterous and faster serving.

Therefore, in this scenario, the RSS space tug brings the $10,000 \mathrm{~kg}$ RST to the RSS for servicing, and an RSS mounted robot will perform the EVA activity of removing and replacing the RST modules. After removal, the module would be transferred inside the RSS through an airlock where IVA astronaut activity would be used to replace just the failed component within the module. Afterwards the repaired module would be passed back through the RSS airlock and be replaced on the RST by the RSS robot.

Consistent with this assumption of improving astronaut efficiency, the robot will be a Supervised Autonomy Robot (SAR). This means that it will basically be pre-programmed but will have a variety of sensors to detect anomalies. In the event of any detected problem the SAR will automatically stop and wait for a ground operator to decide what to do about the situation. Even with no detected anomalies, the SAR would also stop at appropriate intervals to allow ground operators to check and confirm status. Should the robot itself need servicing, the support astronaut who is responsible for RSS operation would perform an EVA.

We will make the not unreasonable assumption that the RSS EVA robot is like the SRMS on the ISS and therefore not as dexterous as an EVA astronaut. The consequence will be that the average mass of the robot replaceable module containing the failed or obsolete components will be $50 \mathrm{~kg}$, i.e. twice the mass of an EVA replaced modular component.

However this mass is not relevant here because we are assuming that, with IVA servicing, the average mass of the component to be replaced within the $50-\mathrm{kg}$ module is only 5 $\mathrm{kg}$.

Therefore the 200 components the RST needs over it 20 year life have a total mass of $1000 \mathrm{~kg}$. Let us also assume that the astronaut is 4 times more efficient doing IVA rather than EVA and can replace a component in two hours rather than one 8-hour workday. Now the 200 component replacements over the 20-year life require 400 hours or about a fifth of a year of astronaut time, which at $20,000 \mathrm{~kg}$ of astronaut logistics per year imposes a $4,000 \mathrm{~kg}$ mass requirement on the RST program for the astronaut time. The total mass requirement for this RST servicing option is $15,000 \mathrm{~kg}(10,000$ for the RST, 1,000 for the modules, and 4,000 for the astronaut time), which gives a FOM of 2 $\mathrm{LSR} / \mathrm{kg}$

This gives as good a FOM as shuttle servicing with free astronaut EVA. However, it still requires the human space program to cover both the development cost of the 100,000 kg RSS and its logistics costs for the period of time that the RST is not being serviced.

\section{Scenario 5 (SAR servicing of the RST)}

Let us next look at what could happen if the RST were robot serviced.

Because robots themselves are complex subsystems that can fail we will add two identical Supervised Autonomy Robots (SAR's) to the RST, each of which is designed to be serviced by the other. Let us assume that this robot servicing system and a docking port increase the RST mass by $50 \%$, i.e. from 10,000 to $15,000 \mathrm{~kg}$. We assume that the launch vehicle performs the rendezvous and docking.

We still assume 1 component failure per year per $1,000 \mathrm{~kg}$, so the robot serviceable RST will need 300 new components over 20 years. However, as described above, a SAR is less dexterous than an EVA astronaut; therefore, the average mass of a replaced module will increase to $50 \mathrm{~kg}$. This means that the $15,000 \mathrm{~kg}$ robot serviceable RST will need $15,000 \mathrm{~kg}$ of replacement modules over its 20 year life. Assuming the same 6 servicing missions as for the STS serviced RST (one every three years), each servicing LV will bring up fifty $50-\mathrm{kg}$ modules, or $2500 \mathrm{~kg}$ of modules.

Based on the above assumptions, a robot serviceable RST requires the same mass of $\mathrm{HW}(30,000 \mathrm{~kg})$ as Scenario 1 (two non-serviceable RST's) and produces the same amount of science. If a more detailed and realistic cost-benefit analysis came to the same conclusion, the expected decision would be to stay with the better understood non-serviceable approach.

\section{Scenario 6 (DTR servicing of the RST)}

Finally, let us assume that effective use can be made of the technology for a Dexterous Tele-operated Robot (DTR). DTR's, or their technological equivalents, are now being used successfully to allow surgery to be performed by a remotely located surgeon, so their dexterity is equivalent to that of an IVA astronaut. 
The time delay for performing such surgery has to be no more than about $0.1 \mathrm{sec}$, which still allows a doctor to be located half way around the world, i.e. $20,000 \mathrm{~km}$ distant. This approach could be accomplished in LEO using a ground operator since LEO is only a few hundred kilometers about ground, which is not significant compared to 20,000 $\mathrm{km}$. However, the GEO TDRSS link could not be used since it is introduces more than $70,000 \mathrm{~km}$ to the length of the signal path. To keep a continuous and low time delay communications link between a ground operator and a LEO DTR would require either a large number of ground stations or use of a LEO communications satellite constellation like IRIDIUM.

To put some numbers behind this option, let us assume that the DTR resides in its own RST-equivalent-mass facility, which will also need its own dual servicing robots since it is a complex facility that will have to have module replacement due to equipment failures and upgrades. Therefore, we assign the DTR facility a mass equivalent to the Scenario 5 SAR serviced RST. This means it has a mass of $15,000 \mathrm{~kg}$ and needs $15,000 \mathrm{~kg}$ of replacement modules over a 20 -year time frame; therefore it represents a $30,000 \mathrm{~kg}$ cost. We also assume the DTR facility is able to rendezvous and dock with the RST.

In this scenario, the servicing robot passes a module from the RST over to a DTR, which is probably enclosed to prevent loss of objects that might come loose. We will assume the DTR is only half as dexterous as an IVA astronaut. In other words, on the average it replaces a $10 \mathrm{~kg}$ component, not a $5 \mathrm{~kg}$ component. Therefore the $200 \mathrm{RST}$ components to be replaced over 20 years amount to 2,000 $\mathrm{kg}$.

We also assume that each component replacement takes the DTR 4 hours rather than two hours needed by the IVA astronaut, so a total of 800 hours of work is needed over 20 years. There are 8760 hours in a year, and even though a ground controlled DTR can presumably work around the clock, we will assume that about half of the time it is involved in its own servicing so only 4000 hours a year of are available for tasks like RST servicing. Therefore over 20 years it would have 80,000 hours for such tasks, and the 800 hours needed by the RST represent $1 \%$ of its total capability. Therefore the RST program is responsible for paying for $300 \mathrm{~kg}$, which is $1 \%$ of the $30,000 \mathrm{~kg}$ cost of the DTR system.

Thus, this solution requires the RST program to pay for a $10,000 \mathrm{~kg}$ RST, $2,000 \mathrm{~kg}$ of replaceable modules, and 300 $\mathrm{kg}$ of DTR facility needs, which is a total of $12,300 \mathrm{~kg}$ of mass. This approach gives a FOM of almost $2.44 \mathrm{LSR} / \mathrm{kg}$, which is not only the best so far, but also has the RST program covering its full share of the DTR costs.

\section{Scenario 7 (IVA Servicing of 100 RST's)}

A first look at the previous scenarios would indicate that there is no mass dependency, since all of the replacement rates and servicing costs are defined to vary linearly with mass. However, some other effects come into play, which serve to create important mass dependencies.

Looking first at the effect of reducing RST mass, there is an obvious limit when the RST mass begins to approach the mass of a replacement module. While more detailed study would be needed to determine the real cross over point, let us assume that the limit is 100 times the average mass of a replacement module. What this implies are minimum RST masses of:

- $5000 \mathrm{~kg}$ for robot servicing,

- $2500 \mathrm{~kg}$ for astronaut EVA servicing

- $\quad 500 \mathrm{~kg}$ for IVA servicing

If we further assumed that half of the RST mass is made up of non-serviceable items such as structure and cable harnesses, this suggests there should be a minimum of about 50 modules to make a serviceable RST worthwhile.

As the RST increases in mass one consequence that comes into play is that it is able to carry a greater portion of the total cost of the RSS. Recall that optimum IVA use of the RSS astronauts required only one fifth of a year of work over 20 years for RST servicing, in other words only $1 \%$ of their time. Thus, if RST servicing were the only task, a twoastronaut crew could service one hundred 10,000-kg RST's, a single massive RST with a mass of $1,000,000 \mathrm{~kg}$, or any combination in between that came to one million $\mathrm{kg}$.

Let us look at the consequences of having 100 RST's and having the RST program carry the full cost of the RSS. At the reference replacement rate of 1 component per $1000 \mathrm{~kg}$ per year, 20 years of use of $1,000,000 \mathrm{~kg}$ of RST's will require 20,000 module replacements.

Using the $5 \mathrm{~kg}$ average module mass for IVA servicing, the RST program will be responsible for $100,000 \mathrm{~kg}$ of modules, which, added to the $1,000,000 \mathrm{~kg}$ of RST and $500,000 \mathrm{~kg}$ for the RSS, comes to a total of $1,600,000 \mathrm{~kg}$.

It would be expected that the 100 RST's would have a variety of designs so that groups will be optimized for different wavelength ranges. A given group of RST's could be used to look at multiple targets simultaneously or to function as a formation flying interferometer. However, we assume that each RST still has an average LSR of 30,000 so that their overall scientific value increases with their mass and they will produce a total LSR of 3,000,000.

Using these assumptions, their FOM of merit is still only 1.88; however, now the RST program is paying the full cost of the RSS development and logistics. 
Scenario 8 (IVA Assembly and Servicing of a Massive Space Telescope)

For this scenario, let us assume that instead of 100 RST's, their $1,000,000 \mathrm{~kg}$ of mass is used to create a single larger telescope, which will be designated the Massive Space Telescope (MST). We will also assume that the mass of a telescope is proportional to the area of its primary mirror. Thus the mass of 100 RST's will be the same as a MST with 100 times the mirror area, or equivalently 10 times the mirror diameter.

Although not directly relevant to this cost-benefit analysis, it is interesting to note the result of trying to assign reasonable numbers to RST and MST PM diameters. Current space telescope technology is represented by the Next Generation Space Telescope (NGST) [2], which has the objective of providing at least 25 sq. meter of primary mirror (PM) area with a total observatory mass of about $5,000 \mathrm{~kg}$. Keeping a constant ratio of PM area to mass means that a $10,000 \mathrm{~kg}$ RST using NGST technology might has have a $50 \mathrm{sq} \mathrm{m}$ PM. To keep the numbers simple let us assume that foreseeable technology improvements increase the ratio of PM area to mass by $50 \%$ so that that a $10,000 \mathrm{~kg}$ RST would have a 75 sq. m PM, which means about a $10 \mathrm{~m}$ diameter PM. At this technology level, the mass of 100 RST's could alternatively yield a MST with a PM diameter of $100 \mathrm{~m}$.

However, another effect comes into play here, and it is that increasing the diameter of a filled aperture telescope provides certain unique advantages in resolution, FOV, sensitivity and speed over a number of smaller aperture telescopes that have the same total PM area. Let us say that this value increase is proportional to the diameter of single telescope relative to the diameter of each of an equivalent mass of separate telescopes. Thus the MST is assumed to have a LSR of $30,000,000$

However, a MST is obviously way too large and too heavy to fit in single $\mathrm{LV}$, so some form of space assembly becomes necessary. Astronaut EVA could be used to construct a 100 $m$ diameter space telescope; however, as stated above, this is not the most effective way of using astronauts. Therefore we will assume that astronaut IVA is used to control DTR's, which (along with SAR's where appropriate) perform the actual EVA assembly of the MST.

For simplification, we ignore the time that the actual construction takes and just look at the 20-year operation benefit and servicing cost. The mass for which the RST program is responsible is the same $1,600,000 \mathrm{~kg}$ as was required for the 100 RST's in Scenario 7, but with the LSR increasing by 10 , the FOM goes up by the same factor to 18.75 .

\section{Scenario 9 (SAR assembly and servicing of the MST)}

If the assembly is to be done by less dexterous supervisedautonomy robots (SAR), then we will assume a more crude construction technique that requires larger and less mass efficient assembly pieces, with the result that the mass of the MST doubles to 2 million $\mathrm{kg}$. We now need 40,000 replacement modules of $50 \mathrm{~kg}$, which adds another 2 million $\mathrm{kg}$, for a total mass requirement of 4 million $\mathrm{kg}$.

The LSR is still $30,000,000$ so the FOM is $7.5 \mathrm{MSR} / \mathrm{kg}$, which is significantly worse than using the combination of astronaut IVA and DTR EVA.

\section{Scenario 10 (DTR assembly and servicing of the MST)}

The only difference between this and Scenario 8 is that ground operators will now control the DTR's so that we can avoid all astronaut related costs. Since a DTR in LEO could be just as effective using ground operators as astronauts, the total assembled mass of MST will still be $1,000,000 \mathrm{~kg}$, but the total mass of the replacement modules doubles to 200,00 $\mathrm{kg}$, because the DTR will do the module replacement rather than the more dexterous IVA astronaut.

Recalling that the DTR facility only needed $1 \%$ of its time to service a $10,000 \mathrm{~kg}$ RST, the MST can be serviced with $100 \%$ of its time. Therefore the total mass requirement of the servicing system is $30,000 \mathrm{~kg}$. This leads to a total mass requirement for this scenario of $1,230,000 \mathrm{~kg}$, which for the LSR of $30,000,000$ gives a FOM of $24.39 \mathrm{LSR} / \mathrm{kg}$.

\section{Scenario 11 (SAR servicing of MST in L2 orbit)}

Let us next look at how the cost models are affected by changes in observatory orbit. An orbit around the Sun-Earth L2 Point is attractive for space telescopes. This L2 point is 1.5 million $\mathrm{km}$ from Earth (four times as far as the Moon) in the anti-sun direction. Due to the approximate balancing of gravitational and centrifugal forces in this region, a satellite can orbit around this $\mathrm{L} 2$ point

A good case can be made that a space observatory in orbit about the Sun-Earth L2 point will have at least twice the scientific productivity of a same mass telescope in LEO. This is because:

The view of many astronomical targets will be blocked in LEO by the earth for half of the time,

- Temperature variations will be harder to control in LEO

- A LEO observatory will have to operate off stored power when sunlight is blocked by the earth

- An L2 observatory can be fairly easily shaded from Sun and Earth light, making it much easier to cool which is needed for IR observations. 
On the basis of the current ratios of space hardware cost to launch cost, choosing L2 over LEO looks cost effective if science return is doubled. A representative overall development cost for spacecraft is $\$ 100 \mathrm{~K}$ per $\mathrm{kg}$, and launch costs to LEO are around $\$ 10 \mathrm{~K}$ per $\mathrm{kg}$. An LV can only launch $1 / 3$ of the mass to $L 2$ that it can to LEO, so launch costs to L2 are effectively $\$ 30 \mathrm{~K}$ per $\mathrm{kg}$. Thus $10,000 \mathrm{~kg}$ in LEO effectively costs $\$ 1.1 \mathrm{~B}$, and the same mass costs $\$ 1.3 \mathrm{~B}$ in L2. Because this is a relatively modest cost increase, we will for purposes of simplification ignore it for now and simply assume that going to L2 doubles the LSR without affecting the cost per unit mass.

For the sake of simplicity we will only look at the effect of putting the MST into L2 orbit because it already has the best FOM. Another consideration that makes the MST appropriate for $\mathrm{L} 2$ is that it will be launched in pieces by multiple LV's and then assembled in orbit. This avoids the limit on mass that available and even projected launch vehicles can place into more distant orbits. The Delta IV Heavy [3], which has not even yet flown, is the most capable launch vehicle that can be expected to be available over the next decade, and it can only send about $8,000 \mathrm{~kg}$ to $\mathrm{L} 2$. Its capacity would have to be about doubled just to put the $15,000 \mathrm{~kg}$ non-serviceable (Scenario 1) variant of the RST into $\mathrm{L} 2$ orbit.

Because an $\mathrm{L} 2$ observatory will be about 1.5 million $\mathrm{km}$ from earth, there will be about a 10 second round trip time delay for getting feedback from any ground generated command due to the finite speed of light. This effectively eliminates the ground operated DTR option described in Scenario 10 , which needs at least about a $10 \mathrm{~Hz}$ control loop. Thus, one effect of distant orbits is to make astronauts more valuable. This is because any situation in a distant orbit requiring quick human feedback, such as dexterous manipulation or possibly contingency operations, can only be accomplished by having an astronaut in close proximity.

However, a SAR can still be effectively used in L2 as it basically autonomous, except for anomalies. Assuming that anomalies do not significantly affect the overall mission, a SAR assembled and serviced MST in LW will double the Scenario 9 FOM to a value of $15 \mathrm{LRS} / \mathrm{kg}$.

\begin{tabular}{|c|c|c|c|c|c|c|c|c|c|}
\hline 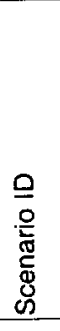 & 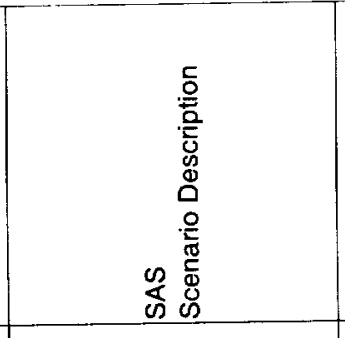 &  & 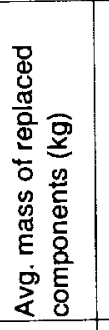 & 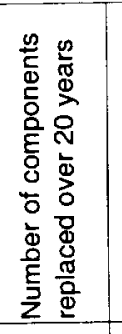 & 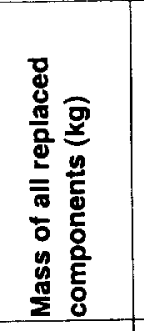 & 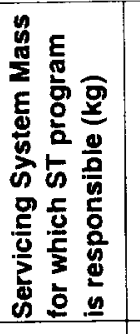 & 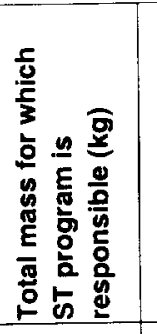 &  & 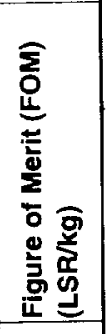 \\
\hline & & & & & & & & & \\
\hline 1 & Non-serviceable RST & 15,000 & 15,000 & 1 & 15,000 & 0 & 30,000 & 30,000 & 1.00 \\
\hline 2 & $\begin{array}{l}\text { STS Astronaut EVA at no } \\
\text { cost to RST }\end{array}$ & 10,000 & 25 & 200 & 5,000 & o & 15,000 & 30,000 & 2.00 \\
\hline 3 & $\begin{array}{l}\text { Astronaut EVA with RST } \\
\text { support of RSS logistics }\end{array}$ & 10,000 & 25 & 200 & 5,000 & 20,000 & 35,000 & 30,000 & 0.86 \\
\hline 4 & $\begin{array}{l}\text { Astronaut IVA with RST } \\
\text { support of RSS logistics }\end{array}$ & 10,000 & 5 & 200 & 1,000 & 4,000 & 15,000 & 30,000 & 2.00 \\
\hline 5 & $\begin{array}{l}\text { Supervised Autonomous } \\
\text { Robot (SAR) }\end{array}$ & 15,000 & 50 & 300 & 15,000 & 0 & 30,000 & 30,000 & 1.00 \\
\hline$\overline{6}$ & $\begin{array}{l}\text { Dexterous Tele-operated } \\
\text { Robot (DTR) }\end{array}$ & 10,000 & 10 & 200 & 2,000 & 300 & 12,300 & 30,000 & $2 . \overline{44}$ \\
\hline 7 & $\begin{array}{l}\text { RSS IVA servicing of } 100 \\
\text { RST's }\end{array}$ & $1,000,000$ & 5 & 20,000 & 100,000 & 500,000 & $1,600,000$ & $3,000,000$ & 1.88 \\
\hline 8 & $\begin{array}{l}\text { RSS IVA assembly and } \\
\text { servicing of MST }\end{array}$ & $1,000,000$ & 5 & 20,000 & 100,000 & 500,000 & $1,600,000$ & $30,000,000$ & 18.75 \\
\hline 9 & $\begin{array}{l}\text { SAR assembly and } \\
\text { servicing of MST }\end{array}$ & $2,000,000$ & 50 & 40,000 & $2,000,000$ & 0 & $4,000,000$ & $30,000,000$ & 7.50 \\
\hline 10 & $\begin{array}{l}\text { DTR assembly and } \\
\text { servicing of MST }\end{array}$ & $1,000,000$ & 10 & 20,000 & 200,000 & 30,000 & $1,230,000$ & $30,000,000$ & 24.39 \\
\hline 11 & $\begin{array}{l}\text { SAR assembly and } \\
\text { servicing of MST at } L 2\end{array}$ & $2,000,000$ & 50 & $40, \overline{000}$ & $2,000,000$ & 0 & $4,000,000$ & $60,000,000$ & 15.00 \\
\hline 12 & $\begin{array}{l}\text { RSS IVA assembly and } \\
\text { servicing of MST at L2 }\end{array}$ & $1,000,000$ & 5 & 20,000 & 100,000 & 500,000 & $1,600,000$ & $60,000,000$ & 37.50 \\
\hline
\end{tabular}


Scenario 12 (RSS IVA servicing of MST at L2

Like the previous SAR scenario, this simply doubles the equivalent (Scenario 8) LEO FOM from 18.75 to 37.5 LSR/kg.

Thus, the end result is that the best FOM comes from putting an MST in L2 orbit and using astronaut IVA to service modules, while the EVA for assembly and module exchange is performed by some optimum mix of SAR's and DTR's.

\section{CONCLUSIONS}

As noted, the above cost-benefit model is the result of an initial and limited investigation. It is, therefore, very simplified and not useful in its present form for determining the most cost effective way to carry out any specific mission. However, its results appear consistent with actual experience in SAS, and so this model seems to provide a framework for using sufficiently complex and realistic representations of SAS costs and benefits to get useful results for specific missions.

However, even in its current simplified form the results appear realistic enough to derive useful guidelines to follow in the conceptual design of future missions. Below are some guidelines for each of the five distinct SAS options defined at the beginning of the paper.

1. A non-serviceable space facility looks fairly costeffective relative to any form of SAS, until the target facility gets too large for a single launch vehicle. Then some form of assembly becomes necessary, and the assembly system can be used to provide the servicing capability at little additional cost.

2. If a set of smaller (and single launch vehicle compatible) space facilities can provide approximately the same mission benefits of a larger, equivalent-mass facility that would have to be assembled, then the set of smaller facilities should be more cost effective

3. A rendezvous and docking capability is needed for any form of SAS, but it alone does not seem sufficient for assembling and servicing space observatories, or other space facilities that have such a specialized geometry and design. However, rendezvous and docking does seem to be effective for the initial stages of building something like a space station, which can consist of a number of similar large modules with similar connections.

4. Astronauts are expensive and so they must be used as effectively as possible to maximize their cost effectiveness. Any combination of the following will increase astronaut effectiveness for SAS a. Perform planned astronaut efforts in a well equipped IVA environment where work can be done quickly and with dexterity

b. Use astronaut controlled DTR's for EVA work requiring dexterity

c. Use SAR's for EVA work that does not require dexterity

d. Use astronauts in remote orbits where the ground controlled alternative become less effective due to communication delays

e. Provide astronaut EVA capability, but limit its use to handling anomalies that cannot be otherwise dealt with

f. Minimizing the size, staffing, and logistics of the space station which provides the astronaut support for SAS

g. The program requiring the SAS (the RST program in this paper) can save money by having other programs, such as the human space program, defray astronaut costs.

5. Any of the following will contribute to increasing the cost effectiveness of robot SAS

a. Use robot EVA so the astronaut can work more efficiently in an IVA environment.

b. Have astronauts or ground operators sufficiently near the robot to perform dexterous tasks using high bandwidth teleoperated control (i.e. DTR's).

c. To minimize human workload, use SAR's when dexterity is not needed

d. Use SAR's in distant orbits when establishing astronauts there is not cost effective. An example could be a $30 \mathrm{~m}$ diameter telescope in L2, which might be too small to cost justify a L2 astronaut facility, but too large for a single $\mathrm{LV}$.

\section{REFERENCES}

[1] http://hubble.nasa.gov/servicing-missions/

[2] http://ngst.gsfc.nasa.gov/

[3] http://www.boeing.com/defense-

space/space/delta/guides.htm

\section{BIOGRAPHY}

Lloyd Purves is an engineer at NASA's Goddard Space Flight Center (GSFC). His work at GSFC has been in the areas of satellite data analysis; computer aided design, manufacturing, modeling and simulation; and robotics. He headed the project to develop the Robot Operated Materials Processing System (ROMPS), a GAS-Hitchhiker payload, which flew successfully on STS-64 in 1995. He is currently supporting the Next Generation Space Telescope (NGST) Project. He has a BSEE from Union College and has done graduate work in finite element analysis. 